\title{
Complex Nonlinear Deformation of Nanometer Intergranular Glassy Films in $\beta-\mathrm{Si}_{3} \mathbf{N}_{4}$
}

\author{
Jun Chen, ${ }^{1,2}$ Lizhi Ouyang, ${ }^{1}$ Paul Rulis, ${ }^{1}$ Anil Misra, ${ }^{3}$ and W. Y. Ching ${ }^{1, *}$ \\ ${ }^{1}$ Department of Physics, University of Missouri-Kansas City, Kansas City, Missouri 64110, USA \\ ${ }^{2}$ Institute of Applied Physics and Computational Mathematics, Beijing, China, 100088 \\ ${ }^{3}$ Department of Civil and Mechanical Engineering, School of Computing and Engineering, University of Missouri-Kansas City,
} Kansas City, Missouri 64110, USA

(Received 12 August 2005; published 16 December 2005)

\begin{abstract}
The mechanical properties of a model of Y-doped intergranular glassy film in silicon nitride ceramics are studied by large-scale ab initio modeling. By linking directly to its electronic structure, it is shown that this microstructure has a complex nonlinear deformation under stress and $\mathrm{Y}$ doping significantly enhances the mechanical properties. The calculation of the electrostatic potential across the film supports the space charge model in ceramic microstructures.
\end{abstract}

DOI: 10.1103/PhysRevLett.95.256103

PACS numbers: 68.35.Gy, 71.10.-w, 82.45.Xy

One of the most intriguing phenomena in ceramics is the existence of nanometer thin intergranular glassy films (IGFs) in polycrystalline samples that form when sintered at high temperature [1-4]. The addition of rare earth (RE) elements drastically changes the structure and properties of the IGF $[5,6]$. The IGFs control many of the mechanical properties of sintered ceramics. Since the brittleness of ceramic materials is a critical issue for their applications, it is important to understand the structure and fundamental interactions within IGF and at its interface with the crystal. Unfortunately, well characterized samples are rare and direct experimental measurements on IGFs are difficult or impossible because of their unique structural complexity. Most recent work focuses on the observation of RE ions in the IGF under high resolution electron microscopes [7]. Accurate computational modeling can play a major role in such cases. Both atomistic calculations and continuum theories of elasticity or their combination have been used for studying metallic systems with simple crystal structures [8-11]. In atomistic simulations, first principles calculations are usually limited to small systems that exploit symmetry to reduce the computational burden. At the continuum level of simulations, parameters that enter into standard codes are usually obtained from homogeneous crystalline solids or empirical methods and are either not applicable to complex microstructures, such as IGFs, or do not provide the link between the fundamental electronic structure and mechanical properties. Most assumptions and simplifications employed in these models certainly will not be valid in multicomponent structures of high complexity. In this Letter, we present results of an accurate large-scale simulation on a model of Y-doped IGF in $\beta-\mathrm{Si}_{3} \mathrm{~N}_{4}$. In addition to the mechanical properties, we provide detailed information on the atomic structure and electrostatic potential distribution that are important for the fundamental understanding of structural ceramics and their future applications.

The model structure for the Y-doped IGF (Y-IGF) used in the present study is shown in Fig. 1(a). It has a glassy region of about $1 \mathrm{~nm}$ sandwiched between crystalline (0001) surfaces of $\beta-\mathrm{Si}_{3} \mathrm{~N}_{4}$ and is periodic. It contains a total of 798 atoms with $16 \mathrm{Y}, 60 \mathrm{Si}, 126 \mathrm{O}$, and $36 \mathrm{~N}$ ions in the IGF region. This gives a ratio of $\mathrm{Y} /(\mathrm{O}+\mathrm{N})=0.10$ and a planar concentration of $2.68 \mathrm{Y}$ ions per $(\mathrm{nm})^{2}$, which is close to that in real samples [12]. This model is obtained from the undoped model [13] by replacing $16 \mathrm{Si}$ ions by $16 \mathrm{Y}$ ions and simultaneous substitutions of $16 \mathrm{~N}$ by $\mathrm{O}$ to maintain charge neutrality. To determine the most likely distribution of $\mathrm{Y}$ ions in the glassy region, we designed four substitution patterns for Y-IGF [Fig. 1(b)]: (i) random distribution, (ii) $\mathrm{Y}$ at the interface, (iii) a graded distribution with less $\mathrm{Y}$ in the middle, and (iv) a graded distribution

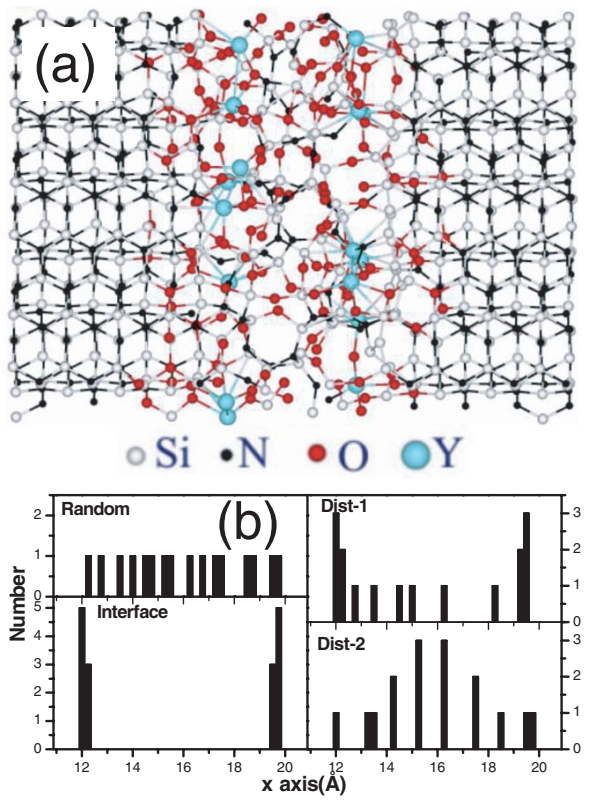

FIG. 1 (color). (a) The ball and stick model of the relaxed YIGF between $\beta-\mathrm{Si}_{3} \mathrm{~N}_{4}$ crystal layers with $\mathrm{Y}$ ions near the interfacial region. Supercell dimensions are $a=30.031 \AA, b=$ $22.783 \AA$, and $c=13.080 \AA$. (b) The four distribution patterns of $16 \mathrm{Y}$ ions in the IGF. 
with more $\mathrm{Y}$ in the middle. These models were fully relaxed using Vienna $a b$ initio simulation package VASP [14]. A two-step process was adopted. First, the volume of the Y-IGF model was optimized, followed by relaxation of all the atoms in the model. We used generalized gradient approximation Perdue-Wang projected augmented wave potentials with one general $k$ point and an energy cutoff of $400 \mathrm{eV}$. The energy and the residual force on each atom were converged to $0.001 \mathrm{eV}$ and $0.01 \mathrm{eV} / \AA$, respectively. In general, it takes 35 to 40 VASP relaxation steps to reach convergence [15]. The interfacial model (ii) [Fig. 1(a)] has the lowest energy and is chosen as the representative model for the evaluation of its mechanical properties. Figure 2 shows the pair distribution function (PDF) of the IGF for both the Y-doped and undoped models. To ensure that all bonds of the atoms at the interfaces are counted, the top layer of the crystalline region is included in the PDF calculation. The amorphous nature of the IGF is obvious. The Si-O and Si-N peaks are very close, and $\mathrm{Y}-\mathrm{O}$ and $\mathrm{Y}-\mathrm{N}$ peaks can be clearly identified. Detailed analysis of the YIGF model shows that of the $60 \mathrm{Si}$ atoms, 44 are fourfold bonded and 16 are threefold bonded. For the $126 \mathrm{O}$ ions, 114 are twofold bonded and 12 are threefold bonded. Of the $36 \mathrm{~N}$ ions, only 8 are threefold bonded and the rest are fourfold bonded. So the glassy structure in this IGF model is far from an ideal network in which $\mathrm{Si}$ is tetrahedrally bonded to either bridging $\mathrm{O}$ ions or threefold bonded $\mathrm{N}$.

The mechanical properties of the Y-IGF model were investigated by theoretical tensile experiments, together with the undoped model. A uniaxial strain was applied in the $x$ direction in small increments with the bulk crystal part fixed. The $y$ and $z$ dimensions of the model were kept constant such that the initial strain components were $\varepsilon_{11} \neq$ 0 , while $\varepsilon_{22}=\varepsilon_{33}=0$. At each strain (referred to the entire periodic model), all atoms in the model were fully relaxed using VASP until the residual force on each atom was reduced to less than $0.01 \mathrm{eV} / \AA$. The relaxed model at a given strain serves as the starting position for the next increment of strain before the model is fully relaxed again. This process is carried on until the total energy (TE) and

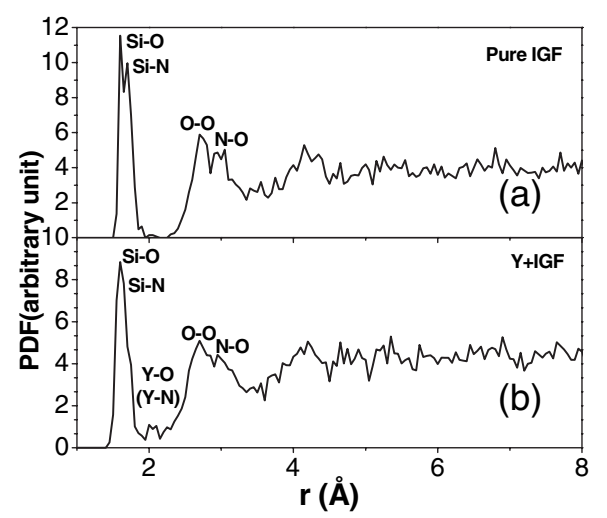

FIG. 2. The PDF of the IGF models: (a) undoped, (b) Y-doped. stress data show that the "sample" is fully fractured. Figure 3 shows the stress components $\sigma_{11}, \sigma_{22}$, and $\sigma_{33}$ and the increase in TE as a function of applied strain $\varepsilon_{11}$. We note that the applied strain in the $x$ direction is mostly accommodated by the IGF part of the model, and the crystalline part experiences almost negligible strain, as shown later in Fig. 4. Thus, the stress-strain response of the model is dominated by the behavior of the IGF. For the Y-doped (undoped) model, $\sigma_{11}$ reaches a maximum of 8.51 (5.94) $\mathrm{GPa}$ at a strain of $2.7 \%(1.3 \%)$. Thus, Y-doping resulted in an increase of the maximum stress and strain by about $43 \%$ and $100 \%$, respectively. For the undoped model, the lateral stresses $\sigma_{22}$ and $\sigma_{33}$ show very little increase in the first few load steps, which correspond to the initial linear portion of the $\sigma_{11}-\varepsilon_{11}$ curve. This stress-strain behavior indicates that the initial atomic structure of the IGF is such that there is negligible coupling between the $x$ and the $y-z$ directions. Such structures are likely to have directional dependent stiffness and tend to be less stiff as opposed to structures with similar composition that exhibit coupling. However, once the $\sigma_{11}-\varepsilon_{11}$ behavior becomes nonlinear, the lateral stresses increase, indicating that the initial atomic structure evolves with loading. As the load carrying capacity of atomic interactions weakens in the $x$ direction, the structure adjusts such that the load is carried through interactions in the $y$ and $z$ directions. For the Ydoped model, the initial structure is stiff in all directions, as indicated by the increase in $\sigma_{22}$ and $\sigma_{33}$ in the early part of loading. As can be seen from separate bond order calculations [15], the toughening exhibited by the Y-doped model could be due to the initial stiffer structure of the Y-IGF. It could also, in part, be attributed to the reduction in surface energy and, consequentially, reduction in residual surface stresses due to the addition of Y. Figure 3(d)
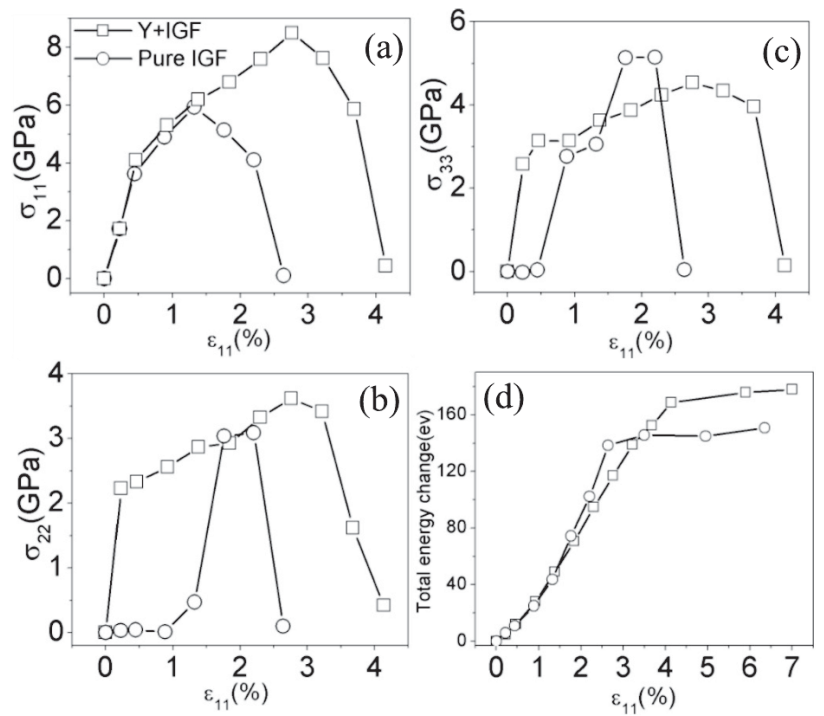

FIG. 3. Stress-strain relationship of the pure and the Y-doped IGF models: (a) $\sigma_{11}$ vs $\varepsilon_{11}$, (b) $\sigma_{22}$ vs $\varepsilon_{11}$, and (c) $\sigma_{33}$ vs $\varepsilon_{11}$. (d) Increase in TE as a function of $\varepsilon_{11}$. 


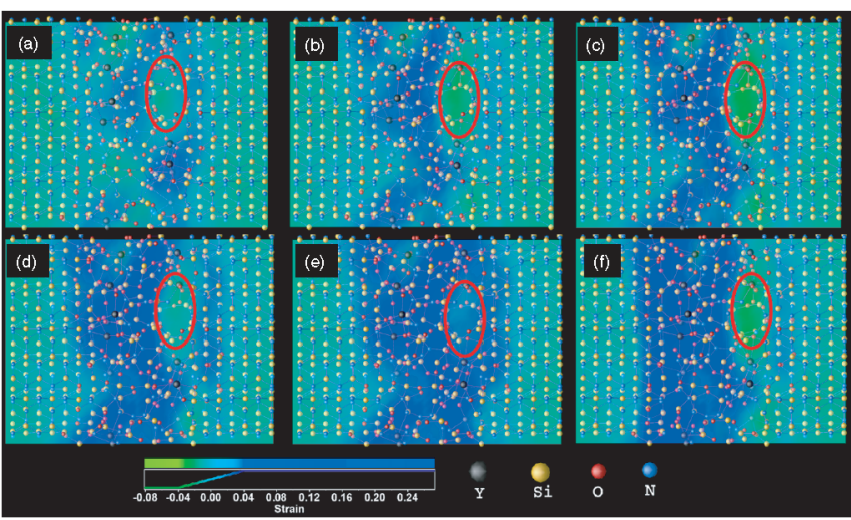

FIG. 4 (color). Local strain field of the Y-IGF as a function of deformation under increasing strain: (a) $0.5 \%$, (b) $0.9 \%$, (c) $2.3 \%$, (d) $2.7 \%$, (e) $3.1 \%$, and (f) $3.7 \%$. The magnitude of the field is indicated by the color bar. The strain field shows a complex pattern as strain increases and is not symmetric at the two sides of the boundaries. Red circles indicate the softer spots in the IGF.

shows the increase in TE between the unstrained model and the fully fractured model which gives the work of separation or fracture energy for the IGF interface. The estimated work of separation per interface for the Y-doped (undoped) IGF is $4.45(4.05) \mathrm{J} / \mathrm{m}^{2}$. It is also noted that the TE continues to rise well beyond the point where the stress reaches the maximum.

To link the nanoscale atomistic structure deformation to the continuum theories, it is instrumental to examine the strain field in the IGF model. In Fig. 4, we show the ball and stick model of Y-IGF under different strains. The elongation of the sample comes almost entirely from the movement of IGF atoms, since they are generally less strongly bonded than those in the bulk crystal. Superimposed on the same diagram is the local strain field obtained from the discrete atomic level data by using an interpolation scheme. The supercell is divided into a $64 \times$ $50 \times 30$ mesh. At each mesh point, a sphere with radius $R_{0}$ is drawn. For atoms within the sphere, the deformation gradient is calculated by assuming a linear deformation field [16]. This approximation can be improved by adding higher order terms [17] in case the deformation gradient changes rapidly within the sphere. The deformation gradient is then used to construct the Green-Lagrange strain tensor [18]. $R_{0}(4.0 \AA)$ is chosen under two considerations: (a) to ensure a minimal distance that guarantees the existence of solutions to the fitting processes used to obtain the local strain properties; (b) to obtain a maximum resolution for the strain field in the IGF region. Too large a $R_{0}$ will average the local variations and obscure the nonlinear behavior of IGF under stress. Also, as seen from Fig. 2, $R_{0}=4.0 \AA$ allows us to potentially consider the next nearest neighbors. The calculated strain $\varepsilon_{11}$ at the mesh points are then plotted in color with the saturation limit set at $\pm 4 \%$ strain, which is the value for complete fracture
[Fig. 3(a)]. The atoms in the bulk crystalline region have very small movement, and most of the applied strain is accommodated by the considerably softer IGF. As the overall strain on the sample is incrementally increased, the local strain field evolves such that locations proximal to the softer spots, such as that circled in red in Fig. 4, attract higher strains. However, the local strain field within IGF is highly heterogeneous, and its evolution as a function of overall change in strain has a complex pattern that cannot be described in simple terms. We also observe that the spatial fluctuation of the strain field in and near the IGF region has a finite length scale ranging over a few angstroms. These observations show that the Cauchy-Born rule [19] typically used to connect the microscopic atomic motion to macroscopic deformation is violated in the IGF and its proximity. As the load progresses, the strain concentration spots tend to coalesce and eventually form a persistent strain localization zone across the IGF, as seen in Fig. 4(d) at the strain of $2.7 \%$ corresponding to the peak stress. At this high strain, the bonds start to break at the interface region first. This is because there are more undercoordinated ions near the interface and because $\mathrm{Y}$ ions, which have longer bonds, reside mostly at the interface. Thus, it is the weakest bonds which have the largest effect on the failure behavior. This behavior is consistent with recent experimental observation of intergranular cracks in sintered $\mathrm{Si}_{3} \mathrm{~N}_{4}$ using TEM and a high-precision microindentor [20]. At very high strain when the IGF is almost completely fractured, it is also interesting to observe that the atoms near the interface rebound towards the crystalline region, as indicated by the small compressive strain proximal to the encircled region in Fig. 4(f).

The linear elastic constants $C_{i j}$ of the complete model, including the crystalline and the IGF, were calculated from the stress to strain ratio when a small lattice deformation $\delta$ was applied in the $x$ and $y$ directions followed by full relaxation. For Y-IGF, $C_{11}=258.5 \mathrm{GPa}, C_{12}=92.1 \mathrm{GPa}$, and $C_{44}=110.4 \mathrm{GPa}$. For the undoped IGF, $C_{11}=$ $215.7 \mathrm{GPa}, \quad C_{12}=66.1 \mathrm{GPa}$, and $C_{44}=105.6 \mathrm{GPa}$. From $C_{i j}$, the bulk modulus $B$, Young's modulus $Y$, shear modulus $G$ (same as $C_{44}$ ), and the Poisson ratio $\nu$ can be approximately derived. For Y-IGF (undoped IGF), $B=147.5$ (115.7) GPa, $Y=208.5$ (186.1) GPa, and $\gamma=$ $0.26(0.23)$. These bulk elastic parameters all indicate that Y-IGF has better mechanical properties than the undoped one, consistent with the larger fracture energy and maximum stress. When Y ions are introduced as network modifiers, they tend to reduce the overall distortion of the bonds, thereby improving the mechanical properties. In particular, we found that the average (minimum) Si-O and $\mathrm{Si}-\mathrm{N}$ bond lengths in the IGF region were reduced from 1.64 (1.45) and $1.72(1.48) \AA$ in the undoped model to 1.63 (1.44) and 1.68 (1.47) $\AA$ in the Y-IGF, respectively. This resulted in an increase in their bond strength. Although Y-O and Y-N bonds are generally weaker than $\mathrm{Si}-\mathrm{O}$ and $\mathrm{Si}-\mathrm{N}$ bonds, the 


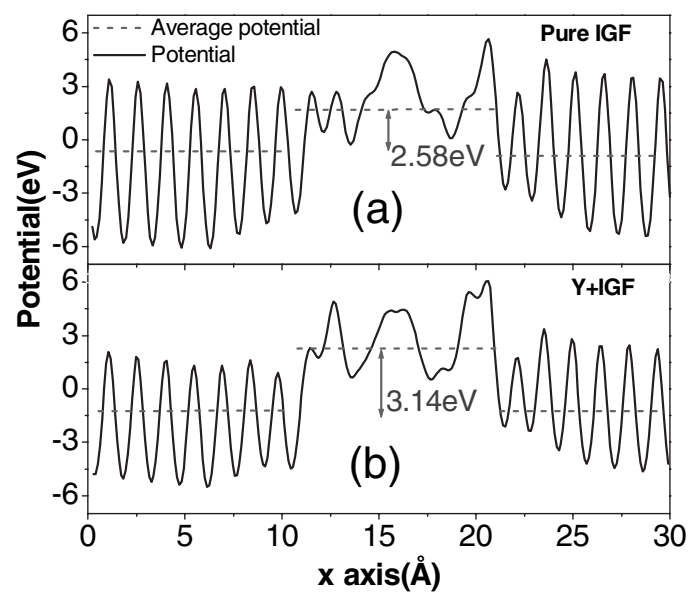

FIG. 5. Electrostatic potential across the IGF at zero strain for (a) pure and (b) Y-doped IGF. The dashed lines show the averaged potential of the $y-z$ plane for the bulk and the IGF regions. $\Delta V$ is obtained from the difference of the dashed lines.

effect is more than compensated for by the increase in bond strength of the shortened $\mathrm{Si}-\mathrm{O}$ and $\mathrm{Si}-\mathrm{N}$ bonds. Thus, atomic relaxation at the interface due to $\mathrm{Y}$ doping may have reduced the interfacial surface tension, which may be an additional factor for the different behavior of lateral stresses in the Y-doped model and undoped models discussed earlier.

In electroceramics, the notion of the space charge model at a grain boundary or other microstructures has been around for quite some time $[21,22]$. No direct evidence has ever been produced to show the existence of such charged layers. Only indirect evidence from impedance types of measurements have been used to explain transport properties in doped ceramics. Here we provide direct evidence for the validity of the space charge model through the calculation of the electrostatic potential $V(\mathbf{r})$ across the IGF. $V(\mathbf{r})$ is obtained from the electronic charge density distribution $\rho(\mathbf{r})$ through Poisson's equation. Figure 5 shows the calculated electrostatic potential difference $\Delta V$ between the bulk and the IGF averaged over the $y-z$ plane for both undoped and Y-doped models. Y doping has increased $\Delta V$ from $2.58 \mathrm{eV}$ in the undoped case to $3.14 \mathrm{eV}$. $\Delta V$ is also found to be insensitive to the applied strain. $\Delta V$ arises from the differences in the dielectric constants of the bulk crystal and the IGF because of their differences in structure and chemical species. Different RE doping changes the dielectric constant of the IGF and $\Delta V$. These are the key parameters in the estimation of the London dispersion force in terms of the Hamaker constants between different layers of materials with different dielectric constants [23] and have been used to predict the equilibrium film thickness of IGF in ceramics [23,24]. The information obtained from the present atomic level electronic structure calculation may, therefore, be used to further investigate the fundamental issue of the existence of IGF in ceramics as well as their mechanical properties. It can be potentially incorporated in other computational schemes such as the quasicontinuum model [25] for simulations on much larger systems.

This research was supported by the U.S. Department of Energy (DOE) under Grant No. DE-FG02-84DR45170 and by NSF Grant No. DMR-00162 in collaboration with the NANOAM Project of EU-CODIS (G5RD-CT-200100586). This research used resources of the NERSC supported by the Office of Science of DOE under Contract No. DE-AC03-76SF00098.

*Electronic address: chingw@umkc.edu

[1] Tailoring of Mechanical Properties of $\mathrm{Si}_{3} \mathrm{~N}_{4}$ Ceramics, edited by M.J. Hoffmann and G. Petzow, NATO ASI Series E, Appl. Sci. Vol. 276 (Kluwer Academic, Dordrecht, 1994).

[2] F. F. Lange, B. I. Davis, and M. G. Metcalf, J. Mater. Sci. 18, 1497 (1983).

[3] H.-J. Kleebe et al., J. Am. Ceram. Soc. 76, 1969 (1993).

[4] I. Tanaka et al., J. Am. Ceram. Soc. 77, 911 (1994).

[5] M. J. Hoffmann, in Ref. [1], p. 59.

[6] P. F. Becher et al., Acta Mater. 48, 4493 (2000).

[7] N. Shibata et al., Nature (London) 428, 730 (2004); A. Ziegler et al., Science 306, 1768 (2004).

[8] V. B. Shenoy et al., Phys. Rev. Lett. 80, 742 (1998).

[9] E. B. Tadmor, G. S. Smith, N. Burnstein, and E. Kaxiras, Phys. Rev. B 59, 235 (1999).

[10] J. Li et al., Nature (London) 418, 307 (2002).

[11] M. Fago, R. L. Hayes, E. A. Carter, and M. Ortiz, Phys. Rev. B 70, 100102(R) (2004).

[12] E. Y. Sun et al., J. Am. Ceram. Soc. 81, 2831 (1998).

[13] P. Rulis et al., Phys. Rev. B 71, 235317 (2005).

[14] G. Kresse and J. Hafner, Phys. Rev. B 47, 558 (1993); G. Kresse and J. Furthmuller, Comput. Mater. Sci. 6, 15 (1996).

[15] We used the IBM Power3 (Seaborg) at the National Energy Research Scientific Computing Center in Lawrence Berkeley Laboratory to carry out these simulations using 256, 512, and up to 1028 processors at different times.

[16] A. Misra and C. S. Chang, Int. J. Solids Struct. 30, 2547 (1993).

[17] C. S. Chang, H. Askes, and L. J. Sluys, Eng. Fract. Mech. 69, 1907 (2002).

[18] Y.C. Fung, Foundations of Solid Mechanics (PrenticeHall, Englewood Cliffs, NJ, 1965).

[19] M. Born and K. Huang, Dynamical Theory of Crystal Lattices (Clarendon, Oxford, 1956).

[20] S. Ii et al., Philos. Mag. 84, 2767 (2004).

[21] W. D. Kingery, J. Am. Ceram. Soc. 57, 1 (1974).

[22] J. A. S. Ikeda and Y. M. Chiang, J. Am. Ceram. Soc. 76, 2437 (1993).

[23] R. H. French et al., Acta Mater. 46, 2271 (1998).

[24] D. R. Clark, J. Am. Ceram. Soc. 70, 15 (1987).

[25] E. B. Tadmor, M. Ortiz, and R. Phillips, Philos. Mag. A 73, 1529 (1996). 